\title{
Uninorms which are neither conjunctive nor disjunctive in interval-valued fuzzy set theory
}

\author{
Glad Deschrijver \\ Fuzziness and Uncertainty Modelling Research Unit \\ Department of Applied Mathematics and Computer Science \\ Ghent University, Krijgslaan 281 (S9), 9000 Gent, Belgium
}

\begin{abstract}
Uninorms are a generalization of t-norms and t-conorms for which the neutral element is an element of $[0,1]$ which is not necessarily equal to 0 (as for t-norms) or 1 (as for t-conorms). Uninorms on the unit interval are either conjunctive or disjunctive, i.e. they aggregate the pair $(0,1)$ to either 0 or 1 . In real-life applications, this kind of aggregation may be counter-intuitive. Interval-valued fuzzy set theory and Atanassov's intuitionistic fuzzy set theory are extensions of fuzzy set theory which allows to model uncertainty about the membership degrees. In these theories there exist uninorms which are neither conjunctive nor disjunctive. In this paper we study such uninorms more deeply and we investigate the structure of these uninorms. We also give several examples of uninorms which are neither conjunctive nor disjunctive.
\end{abstract}

Key words: interval-valued fuzzy set, uninorm, conjunctive, disjunctive

\section{Introduction}

Interval-valued fuzzy set theory $[13,16]$ is an extension of fuzzy set theory in which to each element of the universe a closed subinterval of the unit interval is assigned which approximates the unknown membership degree (using interval-valued fuzzy sets is not always the best approach to deal with uncertainty, see [9] for more information). Another extension of fuzzy set theory is intuitionistic fuzzy set theory introduced by Atanassov [1]. In [7] it is shown that intuitionistic fuzzy set theory is equivalent to interval-valued fuzzy set

Email address: Glad.Deschrijver@UGent. be (Glad Deschrijver). 
theory and that both are equivalent to $L$-fuzzy set theory in the sense of Goguen [12] w.r.t. a special lattice $\mathcal{L}^{I}$.

Uninorms are an important generalization of t-norms and t-conorms introduced by Yager and Rybalov [20]. Uninorms allow for a neutral element lying anywhere in the unit interval rather than at one or zero as is the case for tnorms and t-conorms. Uninorms on the unit interval are either conjunctive or disjunctive [20], i.e. they aggregate the pair $(0,1)$ to either 0 or 1 . In real-life applications, this kind of aggregation may be counter-intuitive, e.g. in customer satisfaction modelling, if an aspect of the product receives a negative evaluation and another aspect a positive evaluation, then in general the global evaluation will neither be very negative or very positive, but rather be quite uncertain. This situation can be modelled by using uninorms in Atanassov's intuitionistic fuzzy set theory, which can be neither conjunctive nor disjunctive (see [8]). In this paper we therefore investigate such uninorms more deeply.

\section{The lattice $\mathcal{L}^{I}$}

Definition 1 We define $\mathcal{L}^{I}=\left(L^{I}, \leq_{L^{I}}\right)$, where

$$
\begin{aligned}
& L^{I}=\left\{\left[x_{1}, x_{2}\right] \mid\left(x_{1}, x_{2}\right) \in[0,1]^{2} \text { and } x_{1} \leq x_{2}\right\}, \\
& {\left[x_{1}, x_{2}\right] \leq_{L^{I}}\left[y_{1}, y_{2}\right] \Longleftrightarrow\left(x_{1} \leq y_{1} \text { and } x_{2} \leq y_{2}\right), \text { for all }\left[x_{1}, x_{2}\right],\left[y_{1}, y_{2}\right] \text { in } L^{I} .}
\end{aligned}
$$

Similarly as Lemma 2.1 in [7] it can be shown that $\mathcal{L}^{I}$ is a complete lattice.

Definition $2[13,16]$ An interval-valued fuzzy set on $U$ is a mapping $A: U \rightarrow$ $L^{I}$.

Definition 3 [1] An intuitionistic fuzzy set on $U$ is a set

$$
A=\left\{\left(u, \mu_{A}(u), \nu_{A}(u)\right) \mid u \in U\right\}
$$

where $\mu_{A}(u) \in[0,1]$ denotes the membership degree and $\nu_{A}(u) \in[0,1]$ the non-membership degree of $u$ in $A$ and where for all $u \in U, \mu_{A}(u)+\nu_{A}(u) \leq 1$.

An intuitionistic fuzzy set $A$ on $U$ can be represented by the $\mathcal{L}^{I}$-fuzzy set $A$ given by

$$
\begin{aligned}
A: U & \rightarrow L^{I}: \\
u & \mapsto\left[\mu_{A}(u), 1-\nu_{A}(u)\right], \quad \text { for all } u \in U .
\end{aligned}
$$

In Figure 1 the set $L^{I}$ is shown. Note that to each element $x=\left[x_{1}, x_{2}\right]$ of $L^{I}$ corresponds a point $\left(x_{1}, x_{2}\right) \in \mathbb{R}^{2}$. 


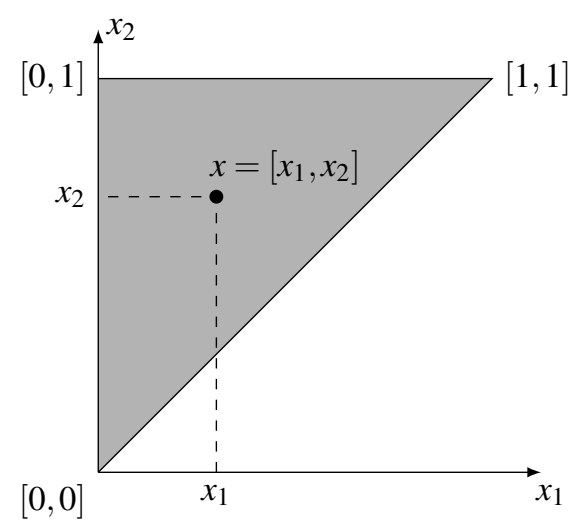

Figure 1. The grey area is $L^{I}$.

In the sequel, if $x \in L^{I}$, then we denote its bounds by $x_{1}$ and $x_{2}$, i.e. $x=$ $\left[x_{1}, x_{2}\right]$. The length $x_{2}-x_{1}$ of the interval $x \in L^{I}$ is called the degree of uncertainty and is denoted by $x_{\pi}$. The smallest and the largest element of $\mathcal{L}^{I}$ are given by $0_{\mathcal{L}^{I}}=[0,0]$ and $1_{\mathcal{L}^{I}}=[1,1]$. Note that, for $x, y$ in $L^{I}, x<_{L^{I}} y$ is equivalent to $x \leq_{L^{I}} y$ and $x \neq y$, i.e. either $x_{1}<y_{1}$ and $x_{2} \leq y_{2}$, or $x_{1} \leq y_{1}$ and $x_{2}<y_{2}$. We define the relation $\ll_{L^{I}}$ by $x \ll_{L^{I}} y \Longleftrightarrow x_{1}<y_{1}$ and $x_{2}<y_{2}$, for $x, y$ in $L^{I}$. If for $x, y$ in $L^{I}$ it holds that either $x_{1}<y_{1}$ and $x_{2}>y_{2}$, or $x_{1}>y_{1}$ and $x_{2}<y_{2}$, then $x$ and $y$ are incomparable w.r.t. $\leq_{L^{I}}$, denoted as $x \|_{L^{I}} y$. We define for further usage the set of degenerate intervals $D=\left\{\left[x_{1}, x_{1}\right] \mid x_{1} \in[0,1]\right\}$.

Note that for any non-empty subset $A$ of $L^{I}$ it holds that

$$
\begin{aligned}
\sup A= & {\left[\sup \left\{x_{1} \mid x_{1} \in[0,1] \text { and }\left(\exists x_{2} \in\left[x_{1}, 1\right]\right)\left(\left[x_{1}, x_{2}\right] \in A\right)\right\},\right.} \\
& \left.\sup \left\{x_{2} \mid x_{2} \in[0,1] \text { and }\left(\exists x_{1} \in\left[0, x_{2}\right]\right)\left(\left[x_{1}, x_{2}\right] \in A\right)\right\}\right] ; \\
\inf A= & {\left[\inf \left\{x_{1} \mid x_{1} \in[0,1] \text { and }\left(\exists x_{2} \in\left[x_{1}, 1\right]\right)\left(\left[x_{1}, x_{2}\right] \in A\right)\right\},\right.} \\
& \left.\inf \left\{x_{2} \mid x_{2} \in[0,1] \text { and }\left(\exists x_{1} \in\left[0, x_{2}\right]\right)\left(\left[x_{1}, x_{2}\right] \in A\right)\right\}\right] .
\end{aligned}
$$

Theorem 4 (Characterization of supremum in $\mathcal{L}^{I}$ ) [6] Let $A$ be an arbitrary non-empty subset of $L^{I}$ and $a \in L^{I}$. Then $a=\sup A$ if and only if

$$
\begin{aligned}
& (\forall x \in A)\left(x \leq_{L^{I}} a\right) \\
& \text { and }\left(\forall \epsilon_{1}>0\right)(\exists z \in A)\left(z_{1}>a_{1}-\epsilon_{1}\right) \\
& \text { and }\left(\forall \epsilon_{2}>0\right)(\exists z \in A)\left(z_{2}>a_{2}-\epsilon_{2}\right) .
\end{aligned}
$$

Definition 5 A t-norm on $\mathcal{L}^{I}$ is a commutative, associative, increasing mapping $\mathcal{T}:\left(L^{I}\right)^{2} \rightarrow L^{I}$ which satisfies $\mathcal{T}\left(1_{\mathcal{L}^{I}}, x\right)=x$, for all $x \in L^{I}$.

A t-conorm on $\mathcal{L}^{I}$ is a commutative, associative, increasing mapping $\mathcal{S}$ : $\left(L^{I}\right)^{2} \rightarrow L^{I}$ which satisfies $\mathcal{S}\left(0_{\mathcal{L}^{I}}, x\right)=x$, for all $x \in L^{I}$.

Definition 6 A negation on $\mathcal{L}^{I}$ is a decreasing mapping $\mathcal{N}: L^{I} \rightarrow L^{I}$ for which $\mathcal{N}\left(0_{\mathcal{L}^{I}}\right)=1_{\mathcal{L}^{I}}$ and $\mathcal{N}\left(1_{\mathcal{L}^{I}}\right)=0_{\mathcal{L}^{I}}$. If $\mathcal{N}(\mathcal{N}(x))=x$, for all $x \in L^{I}$, then 
$\mathcal{N}$ is called involutive.

The mapping $\mathcal{N}_{s}$ defined by $\mathcal{N}_{s}(x)=\left[1-x_{2}, 1-x_{1}\right]$, for all $x \in L^{I}$, is a negation on $\mathcal{L}^{I}$ and is called the standard negation on $\mathcal{L}^{I}$. Note that $\mathcal{N}_{s}(x)=$ $\left[N_{s}\left(x_{2}\right), N_{s}\left(x_{1}\right)\right]$, where $N_{s}$ is the standard negation on $([0,1], \leq)$ given by $N_{s}(a)=1-a$, for all $a \in[0,1]$.

Let $N$ be a negation on $([0,1], \leq)$. Then the mapping $\mathcal{N}_{N}: L^{I} \rightarrow L^{I}$ defined by, for all $x \in L^{I}$,

$$
\mathcal{N}_{N}(x)=\left[N\left(x_{2}\right), N\left(x_{1}\right)\right],
$$

is a negation on $\mathcal{L}^{I}$. Clearly, $\mathcal{N}_{s}=\mathcal{N}_{N_{s}}$.

Theorem 7 [6] A negation $\mathcal{N}$ on $\mathcal{L}^{I}$ is involutive if and only if there exists an involutive negation $N$ on $([0,1], \leq)$ such that $\mathcal{N}=\mathcal{N}_{N}$.

Let $\mathcal{T}$ be a t-norm and $\mathcal{N}$ an involutive negation on $\mathcal{L}^{I}$. Then the mapping $\mathcal{T}_{\mathcal{N}}^{*}:\left(L^{I}\right)^{2} \rightarrow L^{I}$ defined by, for all $x, y$ in $L^{I}, \mathcal{T}_{\mathcal{N}}^{*}(x, y)=\mathcal{N}(\mathcal{T}(\mathcal{N}(x), \mathcal{N}(y)))$, is a t-conorm on $\mathcal{L}^{I}$, called the dual t-conorm of $\mathcal{T}$ w.r.t. $\mathcal{N}$. Similarly the dual t-norm of a t-conorm w.r.t. an involutive negation on $\mathcal{L}^{I}$ is defined.

If for a mapping $f$ on $[0,1]$ and a mapping $F$ on $L^{I}$ it holds that $F([a, a])=$ $[f(a), f(a)]$, for all $a \in[0,1]$, then we say that $F$ is a natural extension of $f$ to $L^{I}$. Note that $F(D) \subseteq D$ if and only if there exists a mapping $f$ on $[0,1]$ such that $F$ is a natural extension of $f$. E.g. $\mathcal{N}_{s}$ is a natural extension of $N_{s}$.

Example 8 Let, for all $x, y$ in $[0,1]$,

$$
\begin{aligned}
T_{W}(x, y) & =\max (0, x+y-1), \\
T_{P}(x, y) & =x y, \\
T_{D}(x, y) & = \begin{cases}\min (x, y), & \text { if } \max (x, y)=1, \\
0, & \text { else, }\end{cases} \\
S_{W}(x, y) & =\min (1, x+y) .
\end{aligned}
$$

Then $T_{W}, T_{P}$ and $T_{D}$ are t-norms, and $S_{W}$ is a t-conorm on $([0,1], \leq)$. Let now, for all $x, y$ in $L^{I}$,

$$
\begin{aligned}
\mathcal{T}_{W}(x, y) & =\left[\max \left(0, x_{1}+y_{1}-1\right), \max \left(0, x_{1}+y_{2}-1, x_{2}+y_{1}-1\right)\right], \\
\mathcal{T}_{P}(x, y) & =\left[x_{1} y_{1}, \max \left(x_{1} y_{2}, x_{2} y_{1}\right)\right], \\
\mathcal{S}_{W}(x, y) & =\left[\min \left(1, x_{1}+y_{2}, x_{2}+y_{1}\right), x_{2}+y_{2}\right] .
\end{aligned}
$$

Then $\mathcal{T}_{W}$ and $\mathcal{T}_{P}$ are t-norms, and $\mathcal{S}_{W}$ is a t-conorm on $\mathcal{L}^{I}$. Furthermore, $\mathcal{T}_{W}$, $\mathcal{T}_{P}$ and $\mathcal{S}_{W}$ are natural extensions of $T_{W}, T_{P}$ and $S_{W}$ respectively.

We will also need the following result and definition (see $[2,14,15,17,18]$ ). 
Theorem 9 Let $\left(T_{\alpha}\right)_{\alpha \in A}$ be a family of t-norms and (]$a_{\alpha}, e_{\alpha}[)_{\alpha \in A}$ be a family of non-empty, pairwise disjoint open subintervals of $[0,1]$. Then the following function $T:[0,1]^{2} \rightarrow[0,1]$ is a t-norm:

$$
T(x, y)= \begin{cases}a_{\alpha}+\left(e_{\alpha}-a_{\alpha}\right) \cdot T_{\alpha}\left(\frac{x-a_{\alpha}}{e_{\alpha}-a_{\alpha}}, \frac{y-a_{\alpha}}{e_{\alpha}-a_{\alpha}}\right), & \text { if }(x, y) \in\left[a_{\alpha}, e_{\alpha}\right]^{2} \\ \min (x, y), & \text { otherwise. }\end{cases}
$$

Definition 10 Let $\left(T_{\alpha}\right)_{\alpha \in A}$ be a family of t-norms and (]$a_{\alpha}, e_{\alpha}[)_{\alpha \in A}$ be a family of non-empty, pairwise disjoint open subintervals of $[0,1]$. The t-norm $T$ defined by (1) is called the ordinal sum of the summands $\left\langle a_{\alpha}, e_{\alpha}, T_{\alpha}\right\rangle, \alpha \in A$, and we will write

$$
T=\left(\left\langle a_{\alpha}, e_{\alpha}, T_{\alpha}\right\rangle\right)_{\alpha \in A}
$$

\section{Uninorms on $\mathcal{L}^{I}$}

The following definition of a uninorm on $\mathcal{L}^{I}$ is a straightforward generalization of the definition of a uninorm on the unit interval introduced by Yager and Rybalov [20,11].

Definition 11 [8] A uninorm on $\mathcal{L}^{I}$ is a commutative, associative, increasing mapping $\mathcal{U}:\left(L^{I}\right)^{2} \rightarrow L^{I}$ for which there exists an $e \in L^{I}$ such that $\mathcal{U}(e, x)=x$, for all $x \in L^{I}$. The element e is called the neutral element of $\mathcal{U}$.

For any uninorm $U$ on the unit interval, there exist increasing bijections $\phi_{e}$ : $[0, e] \rightarrow[0,1]$ and $\psi_{e}:[e, 1] \rightarrow[0,1]$ with increasing inverse, a t-norm $T_{U}$ and a t-conorm $S_{U}$ on $([0,1], \leq)$ such that $[11]$

(i) $\left(\forall(x, y) \in[0, e]^{2}\right)\left(U(x, y)=\phi_{e}^{-1}\left(T_{U}\left(\phi_{e}(x), \phi_{e}(y)\right)\right)\right)$;

(ii) $\left(\forall(x, y) \in[e, 1]^{2}\right)\left(U(x, y)=\psi_{e}^{-1}\left(S_{U}\left(\psi_{e}(x), \psi_{e}(y)\right)\right)\right)$.

Let $\mathcal{U}$ be a uninorm on $\mathcal{L}^{I}$ with neutral element $e \in L^{I}$. We define $E=\{x \mid$ $x \in L^{I}$ and $\left.x \leq_{L^{I}} e\right\}$ and $E^{\prime}=\left\{x \mid x \in L^{I}\right.$ and $\left.x \geq_{L^{I}} e\right\}$. In [8] it is shown that if $e \notin D$, then there does not exist increasing bijections $\Phi_{e}: E \rightarrow L^{I}$ and $\Psi_{e}: E^{\prime} \rightarrow L^{I}$ such that $\Phi_{e}^{-1}$ and $\Psi_{e}^{-1}$ are increasing. On the other hand, if $e \in D \backslash\left\{0_{\mathcal{L}^{I}}, 1_{\mathcal{L}^{I}}\right\}$, then the mappings $\Phi_{e}: E \rightarrow L^{I}$ and $\Psi_{e}: E^{\prime} \rightarrow L^{I}$ defined by, for all $x \in L^{I}$,

$$
\begin{aligned}
& \Phi_{e}(x)=\left[\frac{x_{1}}{e_{1}}, \frac{x_{2}}{e_{1}}\right], \\
& \Psi_{e}(x)=\left[\frac{x_{1}-e_{1}}{1-e_{1}}, \frac{x_{2}-e_{1}}{1-e_{1}}\right] .
\end{aligned}
$$


are increasing bijections for which the inverse is also increasing. As a consequence, the above result can only be extended if $e \in D \backslash\left\{0_{\mathcal{L}^{I}}, 1_{\mathcal{L}^{I}}\right\}$.

From now on, we denote for any t-norm $T$ and t-conorm $S$ on $([0,1], \leq)$, $T_{\phi_{e}}=\phi_{e}^{-1} \circ T \circ\left(\phi_{e} \times \phi_{e}\right)$ and $S_{\psi_{e}}=\psi_{e}^{-1} \circ S \circ\left(\psi_{e} \times \psi_{e}\right)$, where $\times$ denotes the product operation [10]. A similar notation will be used for t-(co)norms and bijections on $\mathcal{L}^{I}$.

Theorem 12 [8] Let $\mathcal{U}$ be a uninorm on $\mathcal{L}^{I}$ with neutral element $e \in D \backslash$ $\left\{0_{\mathcal{L}^{I}}, 1_{\mathcal{L}^{I}}\right\}$. Then:

(i) the mapping $\mathcal{T}_{\mathcal{U}}:\left(L^{I}\right)^{2} \rightarrow L^{I}$ defined by, for all $x, y \in L^{I}$,

$$
\mathcal{T}_{\mathcal{U}}(x, y)=\Phi_{e}\left(\mathcal{U}\left(\Phi_{e}^{-1}(x), \Phi_{e}^{-1}(y)\right)\right)
$$

is a t-norm on $\mathcal{L}^{I}$;

(ii) the mapping $\mathcal{S}_{\mathcal{U}}:\left(L^{I}\right)^{2} \rightarrow L^{I}$ defined by, for all $x, y \in L^{I}$,

$$
\mathcal{S}_{\mathcal{U}}(x, y)=\Psi_{e}\left(\mathcal{U}\left(\Psi_{e}^{-1}(x), \Psi_{e}^{-1}(y)\right)\right)
$$

is a t-conorm on $\mathcal{L}^{I}$.

Similarly as for uninorms on the unit interval, for any uninorm $\mathcal{U}$ on $\mathcal{L}^{I}$ it follows from the monotonicity of $\mathcal{U}$ that

$$
x \leq_{L^{I}} e \leq_{L^{I}} y \Longrightarrow \inf (x, y)=x \leq_{L^{I}} \mathcal{U}(x, y) \leq_{L^{I}} y=\sup (x, y),
$$

for all $x, y$ in $L^{I}$.

These properties show that uninorms are well suited to model human evaluations (e.g. customer satisfaction). Customers which evaluate the performance of all aspects of a certain product high, have a tendency to give the global satisfaction degree an even higher value; on the other hand customers which globally consider the performance of the various aspects as insufficient, will give a low global evaluation. So we observe "reinforcement": a collection of high (low) rates "reinforce" each other and yield a global evaluation rate that is even higher (resp. lower) than each individual rate. If, however, a customer gives high scores only to some aspects and low scores for other aspects, then the global score will in general be located between the lowest and the highest value. This is "compensation". From Theorem 12 it follows that $\left.\mathcal{U}\right|_{E^{2}}$ behaves like a t-norm, in particular $\mathcal{U}(x, y) \leq_{L^{I}} \inf (x, y)$, for all $x, y$ in $E$. On the other hand, $\left.\mathcal{U}\right|_{E^{\prime 2}}$ behaves like a t-conorm, so $\mathcal{U}(x, y) \geq_{L^{I}} \sup (x, y)$, for all $x, y$ in $E^{\prime}$. Finally, if $x \leq_{L^{I}} e$ and $y \geq_{L^{I}} e$ (or conversely), then $\mathcal{U}(x, y)$ is a number between $\inf (x, y)$ and $\sup (x, y)$. So, clearly, uninorms show a reinforcing behaviour on $E^{2}$ and $E^{\prime 2}$, and a compensating behaviour on $E \times E^{\prime}$ and $E^{\prime} \times E$ (see $[3,19,5,4]$ for more details). 
For uninorms on the unit interval, however, $U(0,1)$ can only have two values: 0 or 1 (see [11]). In the first case the uninorm is called "conjunctive" and in the second case "disjunctive". However, in both cases the compensatory behaviour of the uninorm is violated. For uninorms on $\mathcal{L}^{I}$ we have the following property.

Theorem 13 [8] Let $\mathcal{U}$ be a uninorm on $\mathcal{L}^{I}$ with neutral element $e \in D \backslash$ $\left\{0_{\mathcal{L}^{I}}, 1_{\mathcal{L}^{I}}\right\}$. Then either $\mathcal{U}\left(0_{\mathcal{L}^{I}}, 1_{\mathcal{L}^{I}}\right)=0_{\mathcal{L}^{I}}$ or $\mathcal{U}\left(0_{\mathcal{L}^{I}}, 1_{\mathcal{L}^{I}}\right)=1_{\mathcal{L}^{I}}$ or $\mathcal{U}\left(0_{\mathcal{L}^{I}}, 1_{\mathcal{L}^{I}}\right) \|_{L^{I}}$ e.

Hence uninorms on $\mathcal{L}^{I}$ are not necessarily conjunctive or disjunctive. It is possible that a uninorm on $\mathcal{L}^{I}$ shows compensatory behaviour between $0_{\mathcal{L}^{I}}$ and $1_{\mathcal{L}^{I}}$. If one aspect of a product has a very negative evaluation $\left(0_{\mathcal{L}^{I}}\right)$ and another aspect is very positively evaluated $\left(1_{\mathcal{L}^{I}}\right)$, then in general it will be very difficult to give a global evaluation of the product, in fact the global evaluation will contain a lot of uncertainty. Therefore it makes more sense to use a uninorm $\mathcal{U}$ for which $\mathcal{U}\left(0_{\mathcal{L}^{I}}, 1_{\mathcal{L}^{I}}\right) \|_{L^{I}}$ e.

\section{Uninorms on $\mathcal{L}^{I}$ which are neither conjunctive nor disjunctive}

In this section we try to obtain more information about the structure of uninorms which are neither conjunctive nor disjunctive by investigating the possible values of $\mathcal{U}(x, y)$ with $x, y$ in $L^{I}$. First we give an example of a uninorm on $\mathcal{L}^{I}$ that is neither conjunctive nor disjunctive, in order to show that such uninorms do exist.

Example 14 Let for all $\left.e_{1} \in\right] 0,1\left[, U_{e_{1}}\right.$ be the uninorm on $([0,1], \leq)$ defined by, for all $x_{1}, y_{1}$ in $[0,1]$,

$$
U_{e_{1}}\left(x_{1}, y_{1}\right)= \begin{cases}\max \left(x_{1}, y_{1}\right), & \text { if } x_{1} \geq e_{1} \text { and } y_{1} \geq e_{1} \\ \min \left(x_{1}, y_{1}\right), & \text { else }\end{cases}
$$

Let now, for all $x, y$ in $L^{I}$,

$$
\mathcal{U}(x, y)=\left[U_{e_{1}}\left(x_{1}, y_{1}\right), 1-U_{1-e_{1}}\left(1-x_{2}, 1-y_{2}\right)\right]
$$

Then $\mathcal{U}$ is a uninorm on $\mathcal{L}^{I}$ with neutral element $e=\left[e_{1}, e_{1}\right]$. Since $\mathcal{U}\left(0_{\mathcal{L}^{I}}, 1_{\mathcal{L}^{I}}\right)=$ $[0,1], \mathcal{U}$ is neither conjunctive nor disjunctive.

In general, if $U_{1}$ is an arbitrary conjunctive uninorm and $U_{2}$ an arbitrary disjunctive uninorm on $([0,1], \leq)$ with $U_{1} \leq U_{2}$, then the mapping

$$
\begin{aligned}
\mathcal{U}:\left(L^{I}\right)^{2} & \rightarrow L^{I}: \\
(x, y) & \mapsto\left[U_{1}\left(x_{1}, y_{1}\right), U_{2}\left(x_{2}, y_{2}\right)\right], \quad \text { for all } x, y \text { in } L^{I},
\end{aligned}
$$

is a uninorm on $\mathcal{L}^{I}$ for which $\mathcal{U}\left(0_{\mathcal{L}^{I}}, 1_{\mathcal{L}^{I}}\right)=[0,1]$. 
Lemma 15 Let $\mathcal{U}$ be a uninorm on $\mathcal{L}^{I}$ with neutral element e $\in D \backslash\left\{0_{\mathcal{L}^{I}}, 1_{\mathcal{L}^{I}}\right\}$. Then, for all $x \in L^{I}$,

(i) either $\mathcal{U}\left(0_{\mathcal{L}^{I}}, x\right)=0_{\mathcal{L}^{I}}$ or $\mathcal{U}\left(0_{\mathcal{L}^{I}}, x\right) \notin E$,

(ii) either $\mathcal{U}\left(1_{\mathcal{L}^{I}}, x\right)=1_{\mathcal{L}^{I}}$ or $\mathcal{U}\left(1_{\mathcal{L}^{I}}, x\right) \notin E^{\prime}$.

PROOF. Let arbitrarily $x \in L^{I}$. Then, using Theorem 12, we obtain for all $y \in E$ that $\mathcal{U}\left(\mathcal{U}\left(x, 0_{\mathcal{L}^{I}}\right), y\right)=\mathcal{U}\left(x, \mathcal{U}\left(0_{\mathcal{L}^{I}}, y\right)\right)=\mathcal{U}\left(x,\left(\mathcal{T}_{\mathcal{U}}\right)_{\Phi_{e}}\left(0_{\mathcal{L}^{I}}, y\right)\right)=$ $\mathcal{U}\left(x, 0_{\mathcal{L}^{I}}\right)$. Assume that $\mathcal{U}\left(x, 0_{\mathcal{L}^{I}}\right) \in E \backslash\left\{0_{\mathcal{L}^{I}}\right\}$, then there exists a $y \in E$ such that $y<_{L^{I}} \mathcal{U}\left(x, 0_{\mathcal{L}^{I}}\right)$. So $\mathcal{U}\left(\mathcal{U}\left(x, 0_{\mathcal{L}^{I}}\right), y\right)=\left(\mathcal{T}_{\mathcal{U}}\right)_{\Phi_{e}}\left(\mathcal{U}\left(x, 0_{\mathcal{L}^{I}}\right), y\right) \leq_{L^{I}} y<_{L^{I}}$ $\mathcal{U}\left(x, 0_{\mathcal{L}^{I}}\right)$, which is a contradiction. Hence $\mathcal{U}\left(x, 0_{\mathcal{L}^{I}}\right)=0_{\mathcal{L}^{I}}$ or $\mathcal{U}\left(x, 0_{\mathcal{L}^{I}}\right) \notin E$. It is shown in a similar way that $\mathcal{U}\left(1_{\mathcal{L}^{I}}, x\right)=1_{\mathcal{L}^{I}}$ or $\mathcal{U}\left(1_{\mathcal{L}^{I}}, x\right) \notin E^{\prime}$.

Lemma 16 Let $\mathcal{U}$ be a uninorm on $\mathcal{L}^{I}$ with neutral element e $\in D \backslash\left\{0_{\mathcal{L}^{I}}, 1_{\mathcal{L}^{I}}\right\}$. If $\mathcal{U}\left(0_{\mathcal{L}^{I}}, 1_{\mathcal{L}^{I}}\right) \|_{L^{I}} e$, then, for all $x \in L^{I}$,

(i) $\mathcal{U}\left(0_{\mathcal{L}^{I}}, x\right) \|_{L^{I}}$ e or $\mathcal{U}\left(0_{\mathcal{L}^{I}}, x\right)=0_{\mathcal{L}^{I}}$,

(ii) $\mathcal{U}\left(1_{\mathcal{L}^{I}}, x\right) \|_{L^{I}}$ e or $\mathcal{U}\left(1_{\mathcal{L}^{I}}, x\right)=1_{\mathcal{L}^{I}}$.

PROOF. Let arbitrarily $x \in L^{I}$. From Lemma 15 it follows that $\mathcal{U}\left(0_{\mathcal{L}^{I}}, x\right)=$ $0_{\mathcal{L}^{I}}$ or $\mathcal{U}\left(0_{\mathcal{L}^{I}}, x\right) \notin E$. If $\mathcal{U}\left(0_{\mathcal{L}^{I}}, x\right) \notin E$, then $\left(\mathcal{U}\left(0_{\mathcal{L}^{I}}, x\right)\right)_{2}>e_{1}$. From $\left(\mathcal{U}\left(0_{\mathcal{L}^{I}}\right.\right.$, $x))_{1} \leq\left(\mathcal{U}\left(0_{\mathcal{L}^{I}}, 1_{\mathcal{L}^{I}}\right)\right)_{1}<e_{1}$ it follows that $\mathcal{U}\left(0_{\mathcal{L}^{I}}, x\right) \|_{L^{I}} e$. The second part is proven in a similar way.

If one aspect of a product has a negative evaluation $x \in L^{I}$ with $x \leq_{L^{I}}$ e and another aspect has a positive evaluation $y \in L^{I}$ with $y \geq_{L^{I}} e$, then the global evaluation will be rather neutral and contain some uncertainty. Therefore it is natural to expect that $\mathcal{U}(x, y) \|_{L^{I}} e$. We investigate for which $x$ and $y$ in $L^{I}$ this is the case.

Lemma 17 Let $\mathcal{U}$ be a uninorm on $\mathcal{L}^{I}$ with neutral element e $\in D \backslash\left\{0_{\mathcal{L}^{I}}, 1_{\mathcal{L}^{I}}\right\}$. Assume that $\mathcal{U}\left(0_{\mathcal{L}^{I}}, 1_{\mathcal{L}^{I}}\right) \|_{L^{I}}$ e.

(i) Let arbitrarily $x \in E$. If $\mathcal{U}\left(1_{\mathcal{L}^{I}}, x\right)=1_{\mathcal{L}^{I}}$, then $\mathcal{U}\left(1_{\mathcal{L}^{I}},\left[x_{1}, y_{2}\right]\right)=1_{\mathcal{L}^{I}}$, for all $y_{2} \in\left[x_{1}, e_{1}\right]$.

(ii) Let arbitrarily $x \in E^{\prime}$. If $\mathcal{U}\left(0_{\mathcal{L}^{I}}, x\right)=0_{\mathcal{L}^{I}}$, then $\mathcal{U}\left(0_{\mathcal{L}^{I}},\left[y_{1}, x_{2}\right]\right)=0_{\mathcal{L}^{I}}$, for all $y_{1} \in\left[e_{1}, x_{2}\right]$.

PROOF. We show the first part, the second part is proven in a similar way. Let $x \in E$ such that $\mathcal{U}\left(1_{\mathcal{L}^{I}}, x\right)=1_{\mathcal{L}^{I}}$. Assume that $\mathcal{U}\left(1_{\mathcal{L}^{I}},\left[x_{1}, x_{1}\right]\right) \|_{L^{I}} e$. We will show that this assumption is incorrect, so from Lemma 16(ii) it will follow 
that $\mathcal{U}\left(1_{\mathcal{L}^{I}},\left[x_{1}, x_{1}\right]\right)=1_{\mathcal{L}^{I}}$ which together with the monotonicity of $\mathcal{U}$ shows the result.

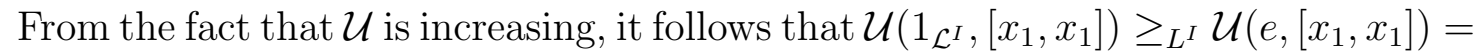
$\left[x_{1}, x_{1}\right]$, so $\left(\mathcal{U}\left(1_{\mathcal{L}^{I}},\left[x_{1}, x_{1}\right]\right)\right)_{1} \geq x_{1}$. On the other hand, from $\mathcal{U}\left(1_{\mathcal{L}^{I}},\left[x_{1}, x_{1}\right]\right) \|_{L^{I}} e$ it follows that $\left(\mathcal{U}\left(1_{\mathcal{L}^{I}},\left[x_{1}, x_{1}\right]\right)\right)_{2} \geq e_{1} \geq x_{2}$ (using the fact that $x \in E$ ). Hence $\mathcal{U}\left(1_{\mathcal{L}^{I}},\left[x_{1}, x_{1}\right]\right) \geq_{L^{I}} x$. We obtain

$$
\begin{aligned}
\mathcal{U}\left(1_{\mathcal{L}^{I}},\left[x_{1}, x_{1}\right]\right) & =\mathcal{U}\left(\mathcal{U}\left(1_{\mathcal{L}^{I}}, 1_{\mathcal{L}^{I}}\right),\left[x_{1}, x_{1}\right]\right) \\
& =\mathcal{U}\left(1_{\mathcal{L}^{I}}, \mathcal{U}\left(1_{\mathcal{L}^{I}},\left[x_{1}, x_{1}\right]\right)\right) \\
& \geq_{L^{I}} \mathcal{U}\left(1_{\mathcal{L}^{I}}, x\right)=1_{\mathcal{L}^{I}} \geq_{L^{I}} e
\end{aligned}
$$

which is a contradiction.

Theorem 18 Let $\mathcal{U}$ be a uninorm on $\mathcal{L}^{I}$ with neutral element $e \in D \backslash$ $\left\{0_{\mathcal{L}^{I}}, 1_{\mathcal{L}^{I}}\right\}$. If $\mathcal{U}\left(0_{\mathcal{L}^{I}}, 1_{\mathcal{L}^{I}}\right) \|_{L^{I}} e$, then

(i) there exists an $\alpha \in D \cap E$ such that (see Figure 2)

- $\mathcal{U}\left(1_{\mathcal{L}^{I}}, x\right) \|_{L^{I}}$ e for all $x \in L^{I}$ satisfying $x_{1}<\alpha_{1}$ and $x_{2} \leq e_{1}$, and

- $\mathcal{U}\left(1_{\mathcal{L}^{I}}, x\right)=1_{\mathcal{L}^{I}}$, for all $x \in L^{I}$ satisfying $x_{1}>\alpha_{1}$,

(ii) there exists a $\beta \in D \cap E^{\prime}$ such that

- $\mathcal{U}\left(0_{\mathcal{L}^{I}}, x\right) \|_{L^{I}}$ e for all $x \in L^{I}$ satisfying $x_{1} \geq e_{1}$ and $x_{2}>\beta_{1}$, and

- $\mathcal{U}\left(0_{\mathcal{L}^{I}}, x\right)=0_{\mathcal{L}^{I}}$, for all $x \in L^{I}$ satisfying $x_{2}<\beta_{1}$.

PROOF. This follows immediately from the previous lemmas and the fact that $\mathcal{U}$ is increasing.

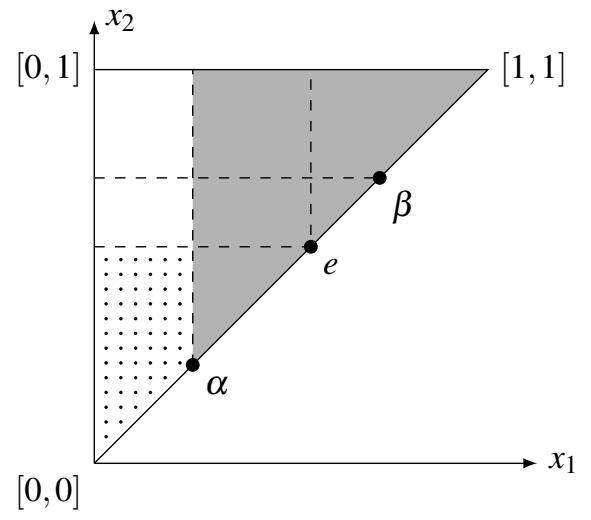

Figure 2. The grey area is the set of elements $x$ for which $\mathcal{U}\left(x, 1_{\mathcal{L}^{I}}\right)=1_{\mathcal{L}^{I}}$, the dotted area is the set of elements $x$ for which $\mathcal{U}\left(x, 1_{\mathcal{L}^{I}}\right) \|_{L^{I}} e$.

Example 19 We give an example of a uninorm which satisfies the results in Theorem 18 for a non-trivial $\alpha$ and $\beta$. Let arbitrarily $e \in D \backslash\left\{0_{\mathcal{L}^{I}}, 1_{\mathcal{L}^{I}}\right\}$, 
$\alpha \in D \cap E \backslash\left\{0_{\mathcal{L}^{I}}, e\right\}$ and $\beta \in D \cap E^{\prime} \backslash\left\{e, 1_{\mathcal{L}^{I}}\right\}$. Let $T_{1 a}$ and $T_{1 b}$ be arbitrary t-norms, $S_{2 a}$ and $S_{2 b}$ arbitrary $t$-conorms on $([0,1], \leq)$, and define

$$
\begin{aligned}
& T_{1}=\left(\left\langle 0, \phi_{e}\left(\alpha_{1}\right), T_{1 a}\right\rangle,\left\langle\phi_{e}\left(\alpha_{1}\right), 1, T_{1 b}\right\rangle\right), \\
& S_{2}=\left(\left\langle 0, \psi_{e}\left(\beta_{1}\right), S_{2 a}\right\rangle,\left\langle\psi_{e}\left(\beta_{1}\right), 1, S_{2 b}\right\rangle\right),
\end{aligned}
$$

using Definition 10 (and using a similar definition for the t-conorm $S_{2}$ ). Let furthermore $T_{2}$ be an arbitrary t-norm and $S_{1}$ an arbitrary $t$-conorm on $([0,1], \leq)$ sucht that $T_{1} \leq T_{2}$ and $S_{1} \leq S_{2}$. Define the mappings $U_{1}:[0,1]^{2} \rightarrow$ $[0,1]$ and $U_{2}:[0,1]^{2} \rightarrow[0,1]$ by, for all $x_{1}, y_{1}, x_{2}, y_{2}$ in $[0,1]$,

$$
\begin{aligned}
& U_{1}\left(x_{1}, y_{1}\right)=\left\{\begin{array}{l}
\left(T_{1}\right)_{\phi_{e}}\left(x_{1}, y_{1}\right), \text { if } \max \left(x_{1}, y_{1}\right) \leq e_{1}, \\
\left(S_{1}\right)_{\psi_{e}}\left(x_{1}, y_{1}\right), \text { if } \min \left(x_{1}, y_{1}\right) \geq e_{1}, \\
1, \quad \text { if }\left(x_{1}>\alpha_{1} \text { and } y_{1}=1\right) \\
\text { or }\left(y_{1}>\alpha_{1} \text { and } x_{1}=1\right), \\
\min \left(x_{1}, y_{1}\right), \text { else, }
\end{array}\right. \\
& U_{2}\left(x_{2}, y_{2}\right)=\left\{\begin{array}{l}
\left(T_{2}\right)_{\phi_{e}}\left(x_{2}, y_{2}\right), \text { if } \max \left(x_{2}, y_{2}\right) \leq e_{1}, \\
\left(S_{2}\right)_{\psi_{e}}\left(x_{2}, y_{2}\right), \text { if } \min \left(x_{2}, y_{2}\right) \geq e_{1}, \\
0, \quad \text { if }\left(x_{2}<\beta_{1} \text { and } y_{2}=0\right) \\
\text { or }\left(y_{2}<\beta_{1} \text { and } x_{2}=0\right), \\
\max \left(x_{2}, y_{2}\right), \text { else, }
\end{array}\right.
\end{aligned}
$$

Then $U_{1}$ is a conjunctive uninorm and $U_{2}$ is a disjunctive uninorm on $([0,1]$, $\leq)$. Indeed, it can be easily verified that $U_{1}$ and $U_{2}$ are increasing in both arguments, commutative and have $e_{1}$ as neutral element. We check the associativity. Let $\left.\left.x_{1} \in\left[0, \alpha_{1}\right], y_{1} \in\right] \alpha_{1}, 1\right]$ and $z_{1}=1$, then, using the fact that from (4) it follows that $U\left(x_{1}, y_{1}\right)=\left(T_{1}\right)_{\phi_{e}}\left(x_{1}, y_{1}\right)=\min \left(x_{1}, y_{1}\right)$ if $y_{1} \leq e_{1}$,

$$
U\left(x_{1}, U\left(y_{1}, z_{1}\right)\right)=U\left(x_{1}, 1\right)=U\left(\min \left(x_{1}, y_{1}\right), z_{1}\right)=U\left(U\left(x_{1}, y_{1}\right), z_{1}\right)
$$

and

$U\left(y_{1}, U\left(x_{1}, z_{1}\right)\right)=U\left(y_{1}, \min \left(x_{1}, 1\right)\right)=U\left(y_{1}, x_{1}\right)=\min \left(U\left(y_{1}, x_{1}\right), 1\right)=U\left(U\left(y_{1}, x_{1}\right), z_{1}\right)$.

The other cases are shown similarly.

The mapping $\mathcal{U}:\left(L^{I}\right)^{2} \rightarrow L^{I}$ defined by, for all $x, y$ in $L^{I}$,

$$
\mathcal{U}(x, y)=\left[U_{1}\left(x_{1}, y_{1}\right), U_{2}\left(x_{2}, y_{2}\right)\right]
$$

is a uninorm on $\mathcal{L}^{I}$ for which $\mathcal{U}\left(0_{\mathcal{L}^{I}}, 1_{\mathcal{L}^{I}}\right)=[0,1]$ and for which the results in Theorem 18 hold for the given $\alpha$ and $\beta$.

From now on $\alpha$ and $\beta$ will be the elements of $\mathcal{L}^{I}$ introduced in Theorem 18. 
Lemma 20 Let $\mathcal{U}$ be a uninorm on $\mathcal{L}^{I}$ with neutral elemente $\in D \backslash\left\{0_{\mathcal{L}^{I}}, 1_{\mathcal{L}^{I}}\right\}$. If $\mathcal{U}\left(0_{\mathcal{L}^{I}}, 1_{\mathcal{L}^{I}}\right) \|_{L^{I}} e$, then for all $x \in E$ and $y \in E^{\prime}$ satisfying $x_{1}<\alpha_{1}$ and $y_{2}>\beta_{1}$ it holds that $\mathcal{U}(x, y) \|_{L^{I}}$.

PROOF. Let $x \in E$ and $y \in E^{\prime}$ such that $x_{1}<\alpha_{1}$ and $y_{2}>\beta_{1}$. Then from Theorem 18 we know that $\mathcal{U}\left(1_{\mathcal{L}^{I}}, x\right) \|_{L^{I}} e$, so $(\mathcal{U}(x, y))_{1} \leq\left(\mathcal{U}\left(1_{\mathcal{L}^{I}}, x\right)\right)_{1} \leq e_{1}$. Similarly, $\mathcal{U}\left(0_{\mathcal{L}^{I}}, y\right) \|_{L^{I}} e$, so $(\mathcal{U}(x, y))_{2} \geq\left(\mathcal{U}\left(0_{\mathcal{L}^{I}}, y\right)\right)_{2} \geq e_{1}$. Hence, $\mathcal{U}(x, y) \|_{L^{I}} e$.

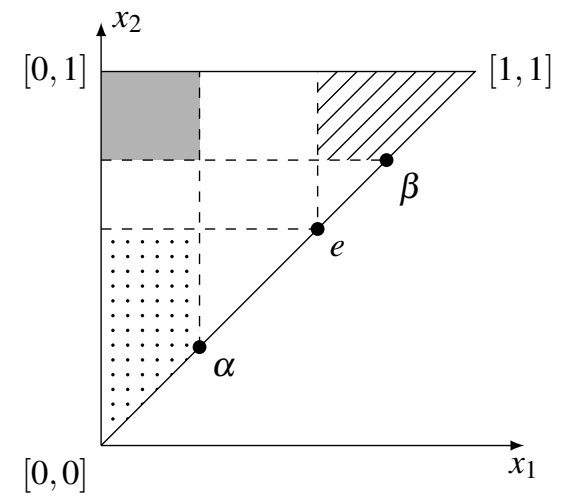

Figure 3. The grey area is the set of possible values of $\mathcal{U}(x, y)$ for $x$ in the dotted area and $y$ in the hashed area.

Theorem 21 Let $\mathcal{U}$ be a uninorm on $\mathcal{L}^{I}$ with neutral element $e \in D \backslash$ $\left\{0_{\mathcal{L}^{I}}, 1_{\mathcal{L}^{I}}\right\}$. If $\mathcal{U}\left(0_{\mathcal{L}^{I}}, 1_{\mathcal{L}^{I}}\right) \|_{L^{I}}$ e, then for all $x \in E$ and $y \in E^{\prime}$ satisfying $x_{1}<\alpha_{1}$ and $y_{2}>\beta_{1}$ it holds that $(\mathcal{U}(x, y))_{1} \leq \alpha_{1}$ and $(\mathcal{U}(x, y))_{2} \geq \beta_{1}$ (see Figure 3).

PROOF. Let $x \in E$ and $y \in E^{\prime}$ such that $x_{1}<\alpha_{1}$ and $y_{2}>\beta_{1}$. Then $\mathcal{U}\left(1_{\mathcal{L}^{I}}, x\right) \|_{L^{I}}$. Assume that $\left(\mathcal{U}\left(1_{\mathcal{L}^{I}}, x\right)\right)_{1}>\alpha_{1}$. Then

$$
\begin{aligned}
\mathcal{U}\left(1_{\mathcal{L}^{I}}, x\right) & =\mathcal{U}\left(\mathcal{U}\left(1_{\mathcal{L}^{I}}, 1_{\mathcal{L}^{I}}\right), x\right) \\
& =\mathcal{U}\left(1_{\mathcal{L}^{I}}, \mathcal{U}\left(1_{\mathcal{L}^{I}}, x\right)\right) \\
& =1_{\mathcal{L}^{I}},
\end{aligned}
$$

where the latter equality follows from the fact that $\left(\mathcal{U}\left(1_{\mathcal{L}^{I}}, x\right)\right)_{1}>\alpha_{1}$ and Theorem 18. This is a contradiction, so we conclude that $\left(\mathcal{U}\left(1_{\mathcal{L}^{I}}, x\right)\right)_{1} \leq \alpha_{1}$. Hence, since $\mathcal{U}$ is increasing, $(\mathcal{U}(x, y))_{1} \leq \alpha_{1}$. In a similar way we find that $(\mathcal{U}(x, y))_{2} \geq \beta_{1}$.

Corollary 22 Let $\mathcal{U}$ be a uninorm on $\mathcal{L}^{I}$ with neutral element $e \in D \backslash$ $\left\{0_{\mathcal{L}^{I}}, 1_{\mathcal{L}^{I}}\right\}$. Assume that $\mathcal{U}\left(0_{\mathcal{L}^{I}}, 1_{\mathcal{L}^{I}}\right) \|_{L^{I}}$ e. 
(i) Let arbitrarily $a=\left[\alpha_{1}, a_{2}\right] \in E$ and $y \in E^{\prime}$. If $\beta_{1}<1$, then

$$
\lim _{\substack{x \rightarrow a \\ x \in E \text { and } x_{1}<\alpha_{1}}}(\mathcal{U}(x, y))_{1}=\alpha_{1} .
$$

(ii) Let arbitrarily $b=\left[b_{1}, \beta_{1}\right] \in E^{\prime}$ and $x \in E$. If $\alpha_{1}>0$, then

$$
\lim _{\substack{y \rightarrow b \\ y \in E^{\prime} \text { and } y_{2}>\beta_{1}}}(\mathcal{U}(x, y))_{2}=\beta_{1} .
$$

In the above, the limits are calculated using on $L^{I}$ the Euclidean metric function $d^{E}(x, y)=\sqrt{\left(x_{1}-y_{1}\right)^{2}+\left(x_{2}-y_{2}\right)^{2}}$, for all $x, y$ in $L^{I}$.

PROOF. Let $a=\left[\alpha_{1}, a_{2}\right] \in E, y \in E^{\prime}$ and $x \in E$ such that $x_{1}<\alpha_{1}$. If $y_{2}>\beta_{1}$, then from Theorem 21 it follows that $(\mathcal{U}(x, y))_{1} \leq \alpha_{1}$. If $y_{2} \leq \beta_{1}$, then there exists a $y^{\prime} \in E^{\prime}$ with $y_{2}^{\prime}>\beta_{1}$ and $y \leq_{L^{I}} y^{\prime}$ (such $y^{\prime}$ exists because $\left.\beta_{1}<1\right)$. So $(\mathcal{U}(x, y))_{1} \leq\left(\mathcal{U}\left(x, y^{\prime}\right)\right)_{1} \leq \alpha_{1}$. On the other hand, since $\mathcal{U}$ is increasing, $(\mathcal{U}(x, y))_{1} \geq(\mathcal{U}(x, e))_{1}=x_{1}$. Combining these inequalities, it is easy to see that

$$
\lim _{\substack{x \rightarrow a \\ x \in E \text { and } x_{1}<\alpha_{1}}}(\mathcal{U}(x, y))_{1}=\alpha_{1} .
$$

The second result is proven in a similar way.

Theorem 23 Let $\mathcal{U}$ be a uninorm on $\mathcal{L}^{I}$ with neutral element $e \in D \backslash$ $\left\{0_{\mathcal{L}^{I}}, 1_{\mathcal{L}^{I}}\right\}$. Assume that $\mathcal{U}\left(0_{\mathcal{L}^{I}}, 1_{\mathcal{L}^{I}}\right) \|_{L^{I}}$ e, $\alpha_{1}>0$ and $\beta_{1}<1$.

(i) For all $x \in E$ and $y \in E^{\prime}$ satisfying $x_{1}>\alpha_{1}$ and $y_{2}>\beta_{1}$ it holds that $\mathcal{U}(x, y) \geq_{L^{I}}\left[\alpha_{1}, \beta_{1}\right]$.

(ii) For all $x \in E$ and $y \in E^{\prime}$ satisfying $x_{1}<\alpha_{1}$ and $y_{2}<\beta_{1}$ it holds that $\mathcal{U}(x, y) \leq_{L^{I}}\left[\alpha_{1}, \beta_{1}\right]$.

PROOF. Let $x \in E$ and $y \in E^{\prime}$ such that $x_{1}>\alpha_{1}$ and $y_{2}>\beta_{1}$. From Theorem 21 and $\alpha_{1}>0$ it follows that $\left(\mathcal{U}\left(0_{\mathcal{L}^{I}}, y\right)\right)_{2} \geq \beta_{1}$. Since $\mathcal{U}$ is increasing, $(\mathcal{U}(x, y))_{2} \geq \beta_{1}$. Using the fact that $\mathcal{U}$ is increasing and Corollary 22 we obtain that $(\mathcal{U}(x, y))_{1} \geq \alpha_{1}$.

\section{The value of $\mathcal{U}\left(0_{\mathcal{L}^{I}}, 1_{\mathcal{L}^{I}}\right)$}

In this section we check which are the possible values for $\mathcal{U}\left(0_{\mathcal{L}^{I}}, 1_{\mathcal{L}^{I}}\right)$ in the case that $\mathcal{U}$ is neither conjunctive nor disjunctive. 
Lemma 24 For any $\alpha \in L^{I}$ and $e \in D \backslash\left\{0_{\mathcal{L}^{I}}, 1_{\mathcal{L}^{I}}\right\}$ such that $\alpha \|_{L^{I}} e, \alpha_{1}>0$ and $\alpha_{2}<1$, there exists an involutive negation $N$ on $([0,1], \leq)$ such that $N\left(\alpha_{1}\right)=\alpha_{2}$ and $N\left(e_{1}\right)=e_{1}$.

PROOF. Define for all $x_{1} \in[0,1]$,

$$
N\left(x_{1}\right)= \begin{cases}1-\frac{1-\alpha_{2}}{\alpha_{1}} x_{1}, & \text { if } x_{1} \in\left[0, \alpha_{1}\right], \\ e_{1}+\frac{\alpha_{2}-e_{1}}{\alpha_{1}-e_{1}}\left(x_{1}-e_{1}\right), & \text { if } x_{1} \in\left[\alpha_{1}, e_{1}\right], \\ e_{1}+\frac{\alpha_{1}-e_{1}}{\alpha_{2}-e_{1}}\left(x_{1}-e_{1}\right), & \text { if } x_{1} \in\left[e_{1}, \alpha_{2}\right], \\ \frac{\alpha_{1}}{1-\alpha_{2}}\left(1-x_{1}\right), & \text { if } x_{1} \in\left[\alpha_{2}, 1\right] .\end{cases}
$$

Then it can be straightforwardly verified that $N$ is an involutive negation with $N\left(\alpha_{1}\right)=\alpha_{2}$ and $N\left(e_{1}\right)=e_{1}$.

Theorem 25 Let $e \in D \backslash\left\{0_{\mathcal{L}^{I}}, 1_{\mathcal{L}^{I}}\right\}, \alpha \in L^{I}, T_{1}$ and $T_{2}$ be t-norms, $S_{1}$ and $S_{2}$ be t-conorms on $([0,1], \leq)$ such that

(i) $\alpha \|_{L^{I}} e$,

(ii) there exist $t$-norms $T_{1 a}$ and $T_{1 b}$ on $([0,1], \leq)$ such that $T_{1}=\left(\left\langle 0, \phi_{e}\left(\alpha_{1}\right), T_{1 a}\right\rangle\right.$, $\left.\left\langle\phi_{e}\left(\alpha_{1}\right), 1, T_{1 b}\right\rangle\right)$,

(iii) there exist $t$-conorms $S_{2 a}$ and $S_{2 b}$ on $([0,1], \leq)$ such that $S_{2}=\left(\left\langle 0, \psi_{e}\left(\alpha_{2}\right), S_{2 a}\right\rangle\right.$, $\left.\left\langle\psi_{e}\left(\alpha_{2}\right), 1, S_{2 b}\right\rangle\right)$

(iv) $T_{1}\left(x_{1}, y_{1}\right) \leq T_{2}\left(x_{1}, y_{1}\right)$ and $S_{1}\left(x_{1}, y_{1}\right) \leq S_{2}\left(x_{1}, y_{1}\right)$, for all $x_{1}, y_{1}$ in $[0,1]$.

Define the mapping $\mathcal{U}:\left(L^{I}\right)^{2} \rightarrow L^{I}$ by, for all $x, y$ in $L^{I}$,

$$
\begin{aligned}
& (\mathcal{U}(x, y))_{1}= \begin{cases}\alpha_{1}, & \text { if }\left(x_{1}<\alpha_{1} \text { and } y_{1} \geq \alpha_{1} \text { and } y_{2}>e_{1}\right) \\
U_{1}\left(x_{1}, y_{1}\right), & \text { or }\left(y_{1}<\alpha_{1} \text { and } x_{1} \geq \alpha_{1} \text { and } x_{2}>e_{1}\right),\end{cases} \\
& (\mathcal{U}(x, y))_{2}= \begin{cases}\alpha_{2}, & \text { if }\left(x_{2}>\alpha_{2} \text { and } y_{2} \leq \alpha_{2} \text { and } y_{1}<e_{1}\right) \\
U_{2}\left(x_{2}, y_{2}\right), & \text { or }\left(y_{2}>\alpha_{2} \text { and } x_{2} \leq \alpha_{2} \text { and } x_{1}<e_{1}\right),\end{cases}
\end{aligned}
$$

where, for all $x_{1}, y_{1}, x_{2}, y_{2}$ in $[0,1]$,

$$
\begin{aligned}
& U_{1}\left(x_{1}, y_{1}\right)= \begin{cases}\left(T_{1}\right)_{\phi_{e}}\left(x_{1}, y_{1}\right), & \text { if } \max \left(x_{1}, y_{1}\right) \leq e_{1}, \\
\left(S_{1}\right)_{\psi_{e}}\left(x_{1}, y_{1}\right), & \text { if } \min \left(x_{1}, y_{1}\right) \geq e_{1}, \\
\min \left(x_{1}, y_{1}\right), & \text { else, }\end{cases} \\
& U_{2}\left(x_{2}, y_{2}\right)= \begin{cases}\left(T_{2}\right)_{\phi_{e}}\left(x_{2}, y_{2}\right), & \text { if } \max \left(x_{2}, y_{2}\right) \leq e_{1}, \\
\left(S_{2}\right)_{\psi_{e}}\left(x_{2}, y_{2}\right), & \text { if } \min \left(x_{2}, y_{2}\right) \geq e_{1}, \\
\max \left(x_{2}, y_{2}\right), & \text { else. }\end{cases}
\end{aligned}
$$


Then $\mathcal{U}$ is a uninorm on $\mathcal{L}^{I}$ with neutral element e for which $\mathcal{U}\left(0_{\mathcal{L}^{I}}, 1_{\mathcal{L}^{I}}\right)=\alpha$.

PROOF. First note that $U_{1}$ and $U_{2}$ are uninorms on $([0,1], \leq)$ with neutral element $e_{1}$. Furthermore $U_{1}$ is conjunctive and $U_{2}$ is disjunctive.

We prove that for all $x, y$ in $L^{I},(\mathcal{U}(x, y))_{1} \leq(\mathcal{U}(x, y))_{2}$, so $\mathcal{U}(x, y)$ is an element of $L^{I}$. Let $x, y$ in $L^{I}$ such that $x_{1}<\alpha_{1}, y_{1} \geq \alpha_{1}$ and $y_{2}>e_{1}$ (the case $y_{1}<\alpha_{1}, x_{1} \geq \alpha_{1}$ and $x_{2}>e_{1}$ is proven similarly). If $x_{2} \geq e_{1}$, then $U_{2}\left(x_{2}, y_{2}\right)=$ $\left(S_{2}\right)_{\psi_{e}}\left(x_{2}, y_{2}\right) \geq e_{1}>\alpha_{1}$. If $x_{2}<e_{1}$, then $U_{2}\left(x_{2}, y_{2}\right)=\max \left(x_{2}, y_{2}\right)=y_{2}>$ $e_{1}>\alpha_{1}$. In a similar way it is shown that $U_{1}\left(x_{1}, y_{1}\right) \leq \alpha_{2}$ for all $x, y$ in $L^{I}$ such that $x_{2}>\alpha_{2} \geq y_{2}$ and $y_{1}<e_{1}$ or such that $y_{2}>\alpha_{2} \geq x_{2}$ and $x_{1}<e_{1}$. Since $\alpha_{1} \leq \alpha_{2}$ and since from (iv) it follows that $U_{1}\left(x_{1}, y_{1}\right) \leq U_{2}\left(x_{2}, y_{2}\right)$, for all $x, y$ in $L^{I}$, we have that $\mathcal{U}(x, y) \in L^{I}$, for all $x, y$ in $L^{I}$.

It is easy to see that $\mathcal{U}$ is commutative.

We show that $\mathcal{U}$ is increasing. Let $x, y, y^{\prime}$ be arbitrary elements of $L^{I}$ such that $y \leq_{L^{I}} y^{\prime}$. Since $U_{1}$ is increasing, in order to prove that the first component of $\mathcal{U}$ is increasing, it suffices to consider the following cases:

- $x_{1}<\alpha_{1}, y_{1}<\alpha_{1}$ or $y_{2} \leq e_{1}, y_{1}^{\prime} \geq \alpha_{1}$ and $y_{2}^{\prime}>e_{1}$ : since $x_{1}<\alpha_{1}<e_{1}$ and either $y_{1}<\alpha_{1}<e_{1}$ or $y_{1} \leq y_{2} \leq e_{1}$, we have that $(\mathcal{U}(x, y))_{1}=U_{1}\left(x_{1}, y_{1}\right)=$ $\left(T_{1}\right)_{\phi_{e}}\left(x_{1}, y_{1}\right) \leq x_{1}<\alpha_{1}=\left(\mathcal{U}\left(x, y^{\prime}\right)\right)_{1}$.

- $x_{1} \geq \alpha_{1}$ and $x_{2}>e_{1}, y_{1}<\alpha_{1}, y_{1}^{\prime} \geq \alpha_{1}$ : we obtain that $(\mathcal{U}(x, y))_{1}=$ $\alpha_{1}$ and $\left(\mathcal{U}\left(x, y^{\prime}\right)\right)_{1}=U_{1}\left(x_{1}, y_{1}^{\prime}\right)$. If $\max \left(x_{1}, y_{1}^{\prime}\right) \leq e_{1}$, then $U_{1}\left(x_{1}, y_{1}^{\prime}\right)=$ $\left(T_{1}\right)_{\phi_{e}}\left(x_{1}, y_{1}^{\prime}\right)$. Since $\min \left(x_{1}, y_{1}^{\prime}\right) \geq \alpha_{1}$, from (ii) it follows that $\left(T_{1}\right)_{\phi_{e}}\left(x_{1}, y_{1}^{\prime}\right) \geq$ $\alpha_{1}$. If $\min \left(x_{1}, y_{1}^{\prime}\right) \geq e_{1}$, then $U_{1}\left(x_{1}, y_{1}^{\prime}\right)=\left(S_{1}\right)_{\psi_{e}}\left(x_{1}, y_{1}^{\prime}\right) \geq e_{1}>\alpha_{1}$. If $\min \left(x_{1}, y_{1}^{\prime}\right)<e_{1}<\max \left(x_{1}, y_{1}^{\prime}\right)$, then $U_{1}\left(x_{1}, y_{1}^{\prime}\right)=\min \left(x_{1}, y_{1}^{\prime}\right) \geq \alpha_{1}$. In all cases we have that $\left(\mathcal{U}\left(x, y^{\prime}\right)\right)_{1} \geq \alpha_{1}=(\mathcal{U}(x, y))_{1}$.

It is shown in a similar way that $(\mathcal{U}(x, y))_{2} \leq\left(\mathcal{U}\left(x, y^{\prime}\right)\right)_{2}$, so $\mathcal{U}$ is increasing in its second argument. From the commutativity of $\mathcal{U}$ it follows that $\mathcal{U}$ is also increasing in its first argument.

We show that $\mathcal{U}$ is associative. Let arbitrarily $x, y, z$ in $L^{I}$. For the following cases we show that $(\mathcal{U}(x, \mathcal{U}(y, z)))_{1}=(\mathcal{U}(y, \mathcal{U}(x, z)))_{1}=(\mathcal{U}(z, \mathcal{U}(x, y)))_{1}$ (the proof for the second component of $\mathcal{U}$ is similar), from the commutativity of $\mathcal{U}$ it will then follow that $\mathcal{U}$ is associative:

- $\max \left(x_{1}, y_{1}, z_{1}\right)<\alpha_{1}$ : we have that $(\mathcal{U}(x, \mathcal{U}(y, z)))_{1}=\left(\mathcal{U}\left(x,\left[U_{1}\left(y_{1}, z_{1}\right)\right.\right.\right.$, $\left.\left.\left.(\mathcal{U}(y, z))_{2}\right]\right)\right)_{1}=U_{1}\left(x_{1}, U_{1}\left(y_{1}, z_{1}\right)\right)$, since $U_{1}\left(y_{1}, z_{1}\right)=\left(T_{1}\right)_{\phi_{e}}\left(y_{1}, z_{1}\right) \leq \min \left(y_{1}\right.$, $\left.z_{1}\right)<\alpha_{1}$. Similarly, we find that $(\mathcal{U}(y, \mathcal{U}(x, z)))_{1}=(\mathcal{U}(z, \mathcal{U}(x, y)))_{1}=$ $U_{1}\left(x_{1}, U_{1}\left(y_{1}, z_{1}\right)\right)$, using the commutativity and associativity of $U_{1}$.

- $\max \left(x_{1}, y_{1}\right)<\alpha_{1} \leq z_{1}$ :

- $z_{2} \leq e_{1}$ : we obtain that $(\mathcal{U}(x, \mathcal{U}(y, z)))_{1}=\left(\mathcal{U}\left(x,\left[U_{1}\left(y_{1}, z_{1}\right),(\mathcal{U}(y, z))_{2}\right]\right)\right)_{1}=$ 
$U_{1}\left(x_{1}, U_{1}\left(y_{1}, z_{1}\right)\right)$, since $U_{1}\left(y_{1}, z_{1}\right)=\left(T_{1}\right)_{\phi_{e}}\left(y_{1}, z_{1}\right) \leq y_{1}<\alpha_{1}$ if $z_{1} \leq e_{1}$, and $U_{1}\left(y_{1}, z_{1}\right)=\min \left(y_{1}, z_{1}\right)=y_{1}<\alpha_{1}$ if $z_{1}>e_{1}$. In a similar way it is shown that $(\mathcal{U}(y, \mathcal{U}(x, z)))_{1}=U_{1}\left(x_{1}, U_{1}\left(y_{1}, z_{1}\right)\right)$. Finally $(\mathcal{U}(z, \mathcal{U}(x, y)))_{1}=$ $\left(\mathcal{U}\left(z,\left[U_{1}\left(x_{1}, y_{1}\right),(\mathcal{U}(x, y))_{2}\right]\right)\right)_{1}=U_{1}\left(z_{1}, U_{1}\left(x_{1}, y_{1}\right)\right)=U_{1}\left(x_{1}, U_{1}\left(y_{1}, z_{1}\right)\right)$, since $U_{1}\left(x_{1}, y_{1}\right)=\left(T_{1}\right)_{\phi_{e}}\left(x_{1}, y_{1}\right) \leq \min \left(x_{1}, y_{1}\right)<\alpha_{1}$.

- $z_{2}>e_{1}$ : we obtain that $(\mathcal{U}(x, \mathcal{U}(y, z)))_{1}=\left(\mathcal{U}\left(x,\left[\alpha_{1},(\mathcal{U}(y, z))_{2}\right]\right)\right)_{1}$. Since either $(\mathcal{U}(y, z))_{2}=\alpha_{2}>e_{1}$ or $(\mathcal{U}(y, z))_{2}=\left(S_{2}\right)_{\psi_{e}}\left(y_{2}, z_{2}\right) \geq \max \left(y_{2}, z_{2}\right) \geq$ $z_{2}>e_{1}$ or $(\mathcal{U}(y, z))_{2}=\max \left(y_{2}, z_{2}\right) \geq z_{2}>e_{1}$, we have that $\left(\mathcal{U}\left(x,\left[\alpha_{1}\right.\right.\right.$, $\left.\left.\left.(\mathcal{U}(y, z))_{2}\right]\right)\right)_{1}=\alpha_{1}$. In a similar way it is shown that $(\mathcal{U}(y, \mathcal{U}(x, z)))_{1}=$ $\alpha_{1}$. Finally $(\mathcal{U}(z, \mathcal{U}(x, y)))_{1}=\left(\mathcal{U}\left(z,\left[U_{1}\left(x_{1}, y_{1}\right),(\mathcal{U}(x, y))_{2}\right]\right)\right)_{1}=\alpha_{1}$, since $U_{1}\left(x_{1}, y_{1}\right)=\left(T_{1}\right)_{\phi_{e}}\left(x_{1}, y_{1}\right) \leq \min \left(x_{1}, y_{1}\right)<\alpha_{1}$.

- $x_{1}<\alpha_{1} \leq \min \left(y_{1}, z_{1}\right)$ :

- $\max \left(y_{2}, z_{2}\right) \leq e_{1}$ : we obtain that $(\mathcal{U}(x, \mathcal{U}(y, z)))_{1}=\left(\mathcal{U}\left(x,\left[U_{1}\left(y_{1}, z_{1}\right)\right.\right.\right.$, $\left.\left.\left.(\mathcal{U}(y, z))_{2}\right]\right)\right)_{1}$. Since $(\mathcal{U}(y, z))_{2}=\left(T_{2}\right)_{\phi_{e}}\left(y_{2}, z_{2}\right) \leq e_{1}$, we have that $(\mathcal{U}(x$, $\left.\left.\left[U_{1}\left(y_{1}, z_{1}\right),(\mathcal{U}(y, z))_{2}\right]\right)\right)_{1}=U_{1}\left(x_{1}, U_{1}\left(y_{1}, z_{1}\right)\right)$. On the other hand, $(\mathcal{U}(y$, $\mathcal{U}(x, z)))_{1}=\left(\mathcal{U}\left(y,\left[U_{1}\left(x_{1}, z_{1}\right),(\mathcal{U}(x, z))_{2}\right]\right)\right)_{1}=U_{1}\left(y_{1}, U_{1}\left(x_{1}, z_{1}\right)\right)$, since neither $y_{1}<\alpha_{1}$ nor both $y_{1} \geq \alpha_{1}$ and $y_{2}>e_{1}$. In a similar way it is shown that $(\mathcal{U}(z, \mathcal{U}(x, y)))_{1}=U_{1}\left(z_{1}, U_{1}\left(x_{1}, y_{1}\right)\right)$.

- $e_{1}<\max \left(y_{2}, z_{2}\right)$ : we obtain that $(\mathcal{U}(x, \mathcal{U}(y, z)))_{1}=\left(\mathcal{U}\left(x,\left[U_{1}\left(y_{1}, z_{1}\right),(\mathcal{U}(y\right.\right.\right.$, $\left.\left.\left.z))_{2}\right]\right)\right)_{1}$. We have the following three possible cases: $U_{1}\left(y_{1}, z_{1}\right)=\left(T_{1}\right)_{\phi_{e}}\left(y_{1}\right.$, $\left.z_{1}\right) \geq \alpha_{1}$ (using (ii)), $U_{1}\left(y_{1}, z_{1}\right)=\left(S_{1}\right)_{\psi_{e}}\left(y_{1}, z_{1}\right) \geq e_{1}>\alpha_{1}$, or $U_{1}\left(y_{1}, z_{1}\right)=$ $\min \left(y_{1}, z_{1}\right) \geq \alpha_{1}$. Furthermore, either $(\mathcal{U}(y, z))_{2}=\alpha_{2}>e_{1}$ or $(\mathcal{U}(y, z))_{2}=$ $\left(S_{2}\right)_{\psi_{e}}\left(y_{2}, z_{2}\right) \geq \max \left(y_{2}, z_{2}\right)>e_{1}$ or $(\mathcal{U}(y, z))_{2}=\max \left(y_{2}, z_{2}\right)>e_{1}$. So $\left(\mathcal{U}\left(x,\left[U_{1}\left(y_{1}, z_{1}\right),(\mathcal{U}(y, z))_{2}\right]\right)\right)_{1}=\alpha_{1}$. For $(\mathcal{U}(y, \mathcal{U}(x, z)))_{1}$, we consider the following two cases:

$* z_{2}>e_{1}$ : in this case, we have $(\mathcal{U}(y, \mathcal{U}(x, z)))_{1}=\left(\mathcal{U}\left(y,\left[\alpha_{1},(\mathcal{U}(x, z))_{2}\right]\right)\right)_{1}$ $=U_{1}\left(y_{1}, \alpha_{1}\right)$. We obtain that either $U_{1}\left(y_{1}, \alpha_{1}\right)=\left(T_{1}\right)_{\phi_{e}}\left(y_{1}, \alpha_{1}\right)=\alpha_{1}$ (using (ii) and the fact that $\left.y_{1} \geq \alpha_{1}\right)$ or $U_{1}\left(y_{1}, \alpha_{1}\right)=\min \left(y_{1}, \alpha_{1}\right)=\alpha_{1}$.

$* z_{2} \leq e_{1}$ : in this case, it necessarily holds that $y_{2}>e_{1}\left(\operatorname{since} \max \left(y_{2}, z_{2}\right)\right.$ $\left.>e_{1}\right)$. We obtain $(\mathcal{U}(y, \mathcal{U}(x, z)))_{1}=\left(\mathcal{U}\left(y,\left[U_{1}\left(x_{1}, z_{1}\right),(\mathcal{U}(x, z))_{2}\right]\right)\right)_{1}$. We have that either $U_{1}\left(x_{1}, z_{1}\right)=\left(T_{1}\right)_{\phi_{e}}\left(x_{1}, z_{1}\right) \leq x_{1}<\alpha_{1}$ or $U_{1}\left(x_{1}, z_{1}\right)$ $=\min \left(x_{1}, z_{1}\right) \leq x_{1}<\alpha_{1}$. So $\left(\mathcal{U}\left(y,\left[U_{1}\left(x_{1}, z_{1}\right),(\mathcal{U}(x, z))_{2}\right]\right)\right)_{1}=\alpha_{1}$.

In a similar way, we obtain that $(\mathcal{U}(z, \mathcal{U}(x, y)))_{1}=\alpha_{1}$.

- $\alpha_{1} \leq \min \left(x_{1}, y_{1}, z_{1}\right)$ : we obtain that $(\mathcal{U}(x, \mathcal{U}(y, z)))_{1}=\left(\mathcal{U}\left(x,\left[U_{1}\left(y_{1}, z_{1}\right)\right.\right.\right.$, $\left.\left.\left.(\mathcal{U}(y, z))_{2}\right]\right)\right)_{1}=U_{1}\left(x_{1}, U_{1}\left(y_{1}, z_{1}\right)\right)$, since either $U_{1}\left(y_{1}, z_{1}\right)=\left(T_{1}\right)_{\phi_{e}}\left(y_{1}, z_{1}\right) \geq$ $\alpha_{1}$ (using (ii)) or $U_{1}\left(y_{1}, z_{1}\right)=\left(S_{1}\right)_{\psi_{e}}\left(y_{1}, z_{1}\right) \geq e_{1}>\alpha_{1}$ or $U_{1}\left(y_{1}, z_{1}\right)=$ $\min \left(y_{1}, z_{1}\right) \geq \alpha_{1}$. Similarly, we find that $(\mathcal{U}(y, \mathcal{U}(x, z)))_{1}=(\mathcal{U}(z, \mathcal{U}(x, y)))_{1}=$ $U_{1}\left(x_{1}, U_{1}\left(y_{1}, z_{1}\right)\right)$.

We obtain that $\left(\mathcal{U}\left(0_{\mathcal{L}^{I}}, 1_{\mathcal{L}^{I}}\right)\right)_{1}=\alpha_{1}$, if $0<\alpha_{1}$ (since $1 \geq \alpha_{1}$ and $\left.1>e_{1}\right)$, and $\left(\mathcal{U}\left(0_{\mathcal{L}^{I}}, 1_{\mathcal{L}^{I}}\right)\right)_{1}=U_{1}(0,1)=\min (0,1)=0$, if $\alpha_{1}=0$. Similarly, we obtain that $\left(\mathcal{U}\left(0_{\mathcal{L}^{I}}, 1_{\mathcal{L}^{I}}\right)\right)_{2}=\alpha_{2}$, so $\mathcal{U}\left(0_{\mathcal{L}^{I}}, 1_{\mathcal{L}^{I}}\right)=\alpha$.

Since neither $e_{1}<\alpha_{1}$ nor $e_{2}>e_{1}$ hold (since $\left.e \in D\right)$, we have that $(\mathcal{U}(e, x))_{1}=$ $U_{1}\left(e_{1}, x_{1}\right)=x_{1}$, for all $x \in L^{I}$. Similarly, $(\mathcal{U}(e, x))_{2}=x_{2}$, for all $x \in L^{I}$, so $e$ 
is the neutral element of $\mathcal{U}$.

Theorem 25 shows that for any $e \in D \backslash\left\{0_{\mathcal{L}^{I}}, 1_{\mathcal{L}^{I}}\right\}$ and any $\alpha \in L^{I}$ such that $\alpha \|_{L^{I}} e$, there exists a uninorm $\mathcal{U}$ on $\mathcal{L}^{I}$ with neutral element $e$ such that $\mathcal{U}\left(0_{\mathcal{L}^{I}}, 1_{\mathcal{L}^{I}}\right)=\alpha$.

In the following theorem we show that for most values of $\alpha \in L^{I}$ such that $\alpha \|_{L^{I}} e$, it is even possible to find uninorms satisfying $\mathcal{U}\left(0_{\mathcal{L}^{I}}, 1_{\mathcal{L}^{I}}\right)=\alpha$, which are self-dual.

Theorem 26 Let $e \in D \backslash\left\{0_{\mathcal{L}^{I}}, 1_{\mathcal{L}^{I}}\right\}, \alpha \in L^{I}$, $T$ be a t-norm, $S$ a t-conorm and $N$ a negation on $([0,1], \leq)$ such that

(i) $\alpha \|_{L^{I}}$ e and either $\alpha \|_{L^{I}}[0,1]$ or $\alpha=[0,1]$,

(ii) $N$ is involutive, $N\left(\alpha_{1}\right)=\alpha_{2}$ and $N\left(e_{1}\right)=e_{1}$,

(iii) there exist $t$-norms $T_{a}$ and $T_{b}$ on $([0,1], \leq)$ such that $T=\left(\left\langle 0, \phi_{e}\left(\alpha_{1}\right), T_{a}\right\rangle\right.$, $\left.\left\langle\phi_{e}\left(\alpha_{1}\right), 1, T_{b}\right\rangle\right)$

(iv) $T_{\phi_{e}}\left(x_{1}, y_{1}\right) \leq N\left(S_{\psi_{e}}\left(N\left(x_{1}\right), N\left(y_{1}\right)\right)\right)$, for all $x_{1}, y_{1}$ in $[0,1]$.

Define the mapping $\mathcal{U}:\left(L^{I}\right)^{2} \rightarrow L^{I}$ by, for all $x, y$ in $L^{I}$,

$$
\begin{aligned}
& (\mathcal{U}(x, y))_{1}= \begin{cases}\alpha_{1}, & \text { if }\left(x_{1}<\alpha_{1} \text { and } y_{1} \geq \alpha_{1} \text { and } y_{2}>e_{1}\right) \\
& \text { or }\left(y_{1}<\alpha_{1} \text { and } x_{1} \geq \alpha_{1} \text { and } x_{2}>e_{1}\right), \\
U\left(x_{1}, y_{1}\right), & \text { else },\end{cases} \\
& (\mathcal{U}(x, y))_{2}=N\left(\left(\mathcal{U}\left(\mathcal{N}_{N}(x), \mathcal{N}_{N}(y)\right)\right)_{1}\right) .
\end{aligned}
$$

where, for all $x_{1}, y_{1}$ in $[0,1]$,

$$
U\left(x_{1}, y_{1}\right)= \begin{cases}T_{\phi_{e}}\left(x_{1}, y_{1}\right), & \text { if } \max \left(x_{1}, y_{1}\right) \leq e_{1} \\ S_{\psi_{e}}\left(x_{1}, y_{1}\right), & \text { if } \min \left(x_{1}, y_{1}\right) \geq e_{1} \\ \min \left(x_{1}, y_{1}\right), & \text { else. }\end{cases}
$$

Then $\mathcal{U}$ is a uninorm on $\mathcal{L}^{I}$ with neutral element e for which $\mathcal{U}\left(0_{\mathcal{L}^{I}}, 1_{\mathcal{L}^{I}}\right)=\alpha$ and, for all $x, y$ in $L^{I}$,

$$
\mathcal{U}(x, y)=\mathcal{N}_{N}\left(\mathcal{U}\left(\mathcal{N}_{N}(x), \mathcal{N}_{N}(y)\right)\right)
$$

PROOF. Note that from Lemma 24 it follows for any $\alpha \in L^{I}$ such that $\alpha \|_{L^{I}} e$ and $\alpha \|_{L^{I}}[0,1]$, that there does exist an involutive negation $N$ on $([0,1], \leq)$ such that (ii) holds. If $\alpha=[0,1]$, then clearly there also exists an involutive negation $N$ for which (ii) holds. 
Let $T_{2}=\phi_{e} \circ N \circ S_{\psi_{e}} \circ\left(\left(N \circ \phi_{e}^{-1}\right) \times\left(N \circ \phi_{e}^{-1}\right)\right), S_{2}=\psi_{e} \circ N \circ T_{\phi_{e}} \circ((N \circ$ $\left.\left.\psi_{e}^{-1}\right) \times\left(N \circ \psi_{e}^{-1}\right)\right)$ and $U_{2}=N \circ U \circ(N \times N)$. For all $x_{2}, y_{2}$ in $[0,1]$ we have

$$
\begin{aligned}
U_{2}\left(x_{2}, y_{2}\right)= & N\left(U\left(N\left(x_{2}\right), N\left(y_{2}\right)\right)\right) \\
= & \begin{cases}N\left(T_{\phi_{e}}\left(N\left(x_{2}\right), N\left(y_{2}\right)\right)\right), & \text { if } \min \left(x_{2}, y_{2}\right) \geq e_{1}, \\
N\left(S_{\psi_{e}}\left(N\left(x_{2}\right), N\left(y_{2}\right)\right)\right), & \text { if } \max \left(x_{2}, y_{2}\right) \leq e_{1}, \\
\max \left(x_{2}, y_{2}\right), & \text { else }\end{cases} \\
= & \begin{cases}\left(S_{2}\right)_{\psi_{e}}\left(x_{2}, y_{2}\right), & \text { if } \min \left(x_{2}, y_{2}\right) \geq e_{1}, \\
\left(T_{2}\right)_{\phi_{e}}\left(x_{2}, y_{2}\right), & \text { if } \max \left(x_{2}, y_{2}\right) \leq e_{1}, \\
\max \left(x_{2}, y_{2}\right), & \text { else. }\end{cases}
\end{aligned}
$$

From (iv) it follows that $T\left(x_{1}, y_{1}\right) \leq \phi_{e}\left(N\left(S_{\psi_{e}}\left(N\left(\phi_{e}^{-1}\left(x_{1}\right)\right), N\left(\phi_{e}^{-1}\left(y_{1}\right)\right)\right)\right)\right)=$ $T_{2}\left(x_{1}, y_{1}\right)$ and $S\left(x_{1}, y_{1}\right) \leq \psi_{e}\left(N\left(T_{\phi_{e}}\left(N\left(\psi_{e}^{-1}\left(x_{1}\right)\right), N\left(\psi_{e}^{-1}\left(y_{1}\right)\right)\right)\right)\right)=S_{2}\left(x_{1}, y_{1}\right)$, for all $x_{1}, y_{1}$ in $[0,1]$.

Let $\phi_{1}\left(x_{1}\right)=\frac{x_{1}}{\phi_{e}\left(\alpha_{1}\right)}$ for all $x_{1} \in\left[0, \phi_{e}\left(\alpha_{1}\right)\right], \phi_{2}\left(x_{1}\right)=\frac{x_{1}-\phi_{e}\left(\alpha_{1}\right)}{1-\phi_{e}\left(\alpha_{1}\right)}$ for all $x_{1} \in$ $\left[\phi_{e}\left(\alpha_{1}\right), 1\right], \psi_{1}\left(x_{2}\right)=\frac{x_{2}}{\psi_{e}\left(\alpha_{2}\right)}$ for all $x_{2} \in\left[0, \psi_{e}\left(\alpha_{2}\right)\right]$, and $\psi_{2}\left(x_{2}\right)=\frac{x_{2}-\psi_{e}\left(\alpha_{2}\right)}{1-\psi_{e}\left(\alpha_{2}\right)}$ for all $x_{2} \in\left[\psi_{e}\left(\alpha_{2}\right), 1\right]$. So we have that $\left.T\right|_{\left[0, \phi_{e}\left(\alpha_{1}\right)\right]^{2}}=\phi_{1}^{-1} \circ T_{a} \circ\left(\phi_{1} \times \phi_{1}\right)=\left(T_{a}\right)_{\phi_{1}}$ and similarly $\left.T\right|_{\left[\phi_{e}\left(\alpha_{1}\right), 1\right]^{2}}=\left(T_{b}\right)_{\phi_{2}}$. Let $S_{2 b}=\psi_{2} \circ \psi_{e} \circ N \circ\left(\left(T_{a}\right)_{\phi_{1}}\right)_{\phi_{e}} \circ\left(\left(N \circ \psi_{e}^{-1} \circ\right.\right.$ $\left.\left.\psi_{2}^{-1}\right) \times\left(N \circ \psi_{e}^{-1} \circ \psi_{2}^{-1}\right)\right)=\left(S_{2}\right)_{\psi_{2}^{-1}}$ and $S_{2 a}=\psi_{1} \circ \psi_{e} \circ N \circ\left(\left(T_{b}\right)_{\phi_{2}}\right)_{\phi_{e}} \circ\left(\left(N \circ \psi_{e}^{-1} \circ\right.\right.$ $\left.\left.\psi_{1}^{-1}\right) \times\left(N \circ \psi_{e}^{-1} \circ \psi_{1}^{-1}\right)\right)=\left(S_{2}\right)_{\psi_{1}^{-1}}$. A straightforward calculation shows that $S_{2 a}$ and $S_{2 b}$ are t-conorms on $([0,1], \leq)$. Furthermore, $\left.S_{2}\right|_{\left[\psi_{e}\left(\alpha_{2}\right), 1\right]^{2}}=\left(S_{2 b}\right)_{\psi_{2}}$ and $\left.S_{2}\right|_{\left[0, \psi_{e}\left(\alpha_{2}\right)\right]^{2}}=\left(S_{2 a}\right)_{\psi_{1}}$. For any $x_{2}, y_{2}$ in $[0,1]$ such that $x_{2}<\psi_{e}\left(\alpha_{2}\right)<$ $y_{2}$, we have that $N\left(\psi_{e}^{-1}\left(x_{2}\right)\right)>N\left(\alpha_{2}\right)=\alpha_{1}>N\left(\psi_{e}^{-1}\left(y_{2}\right)\right)$, so $S_{2}\left(x_{2}, y_{2}\right)=$ $\psi_{e}\left(N\left(\min \left(N\left(\psi_{e}^{-1}\left(x_{2}\right)\right), N\left(\psi_{e}^{-1}\left(y_{2}\right)\right)\right)\right)\right)=\max \left(x_{2}, y_{2}\right)$. Hence $S_{2}=\left\langle 0, \psi_{e}\left(\alpha_{2}\right)\right.$, $\left.\left.S_{2 a}\right\rangle,\left\langle\psi_{e}\left(\alpha_{2}\right), 1, S_{2 b}\right\rangle\right)$.

For all $x, y$ in $L^{I}$ we obtain that

$(\mathcal{U}(x, y))_{2}$

$$
\begin{aligned}
& = \begin{cases}N\left(\alpha_{1}\right), & \text { if }\left(N\left(x_{2}\right)<\alpha_{1} \text { and } N\left(y_{2}\right) \geq \alpha_{1} \text { and } N\left(y_{1}\right)>e_{1}\right) \\
N\left(U\left(N\left(x_{2}\right), N\left(y_{2}\right)\right)\right), & \text { else }\left(N\left(y_{2}\right)<\alpha_{1} \text { and } N\left(x_{2}\right) \geq \alpha_{1} \text { and } N\left(x_{1}\right)>e_{1}\right),\end{cases} \\
& = \begin{cases}\alpha_{2}, & \text { if }\left(x_{2}>\alpha_{2} \text { and } y_{2} \leq \alpha_{2} \text { and } y_{1}<e_{1}\right) \\
U_{2}\left(x_{2}, y_{2}\right), & \text { else }\left(y_{2}>\alpha_{2} \text { and } x_{2} \leq \alpha_{2} \text { and } x_{1}<e_{1}\right),\end{cases}
\end{aligned}
$$

From Theorem 25 it follows that $\mathcal{U}$ is a uninorm on $\mathcal{L}^{I}$ with neutral element $e$ for which $\mathcal{U}\left(0_{\mathcal{L}^{I}}, 1_{\mathcal{L}^{I}}\right)=\alpha$. 
From the fact that $(\mathcal{U}(x, y))_{2}=N\left(\left(\mathcal{U}\left(\mathcal{N}_{N}(x), \mathcal{N}_{N}(y)\right)\right)_{1}\right)$, for all $x, y$ in $L^{I}$, and from the involutivity of $N$ (and thus also of $\left.\mathcal{N}_{N}\right)$ it follows that $(\mathcal{U}(x, y))_{1}=$ $N\left(\left(\mathcal{U}\left(\mathcal{N}_{N}(x), \mathcal{N}_{N}(y)\right)\right)_{2}\right)$, for all $x, y$ in $L^{I}$. $\operatorname{So} \mathcal{U}(x, y)=\mathcal{N}_{N}\left(\mathcal{U}\left(\mathcal{N}_{N}(x), \mathcal{N}_{N}(y)\right)\right)$, for all $x, y$ in $L^{I}$.

Example 27 Let arbitrarily $e \in D$ and $\alpha \in L^{I}$ with $\alpha \|_{L^{I}}$ e and $\alpha \|_{L^{I}}[0,1]$. Define for all $x_{1} \in[0,1]$,

$$
N\left(x_{1}\right)= \begin{cases}1-\frac{1-\alpha_{2}}{\alpha_{1}} x_{1}, & \text { if } x_{1} \in\left[0, \alpha_{1}\right], \\ e_{1}+\frac{\alpha_{2}-e_{1}}{\alpha_{1}-e_{1}}\left(x_{1}-e_{1}\right), & \text { if } x_{1} \in\left[\alpha_{1}, e_{1}\right], \\ e_{1}+\frac{\alpha_{1}-e_{1}}{\alpha_{2}-e_{1}}\left(x_{1}-e_{1}\right), & \text { if } x_{1} \in\left[e_{1}, \alpha_{2}\right], \\ \frac{\alpha_{1}}{1-\alpha_{2}}\left(1-x_{1}\right), & \text { if } x_{1} \in\left[\alpha_{2}, 1\right] .\end{cases}
$$

Then $N$ is an involutive negation with $N\left(\alpha_{1}\right)=\alpha_{2}$ and $N\left(e_{1}\right)=e_{1}$. Define $T=\left(\left\langle 0, \phi_{e}\left(\alpha_{1}\right), P\right\rangle,\left\langle\phi_{e}\left(\alpha_{1}\right), 1, \min \right\rangle\right)$, where $P$ is the product $t$-norm on the unit interval. Then for all $\left(x_{1}, y_{1}\right) \in\left[0, e_{1}\right]^{2}$,

$$
T_{\phi_{e}}\left(x_{1}, y_{1}\right)= \begin{cases}\frac{1}{\alpha_{1}} x_{1} y_{1}, & \text { if }\left(x_{1}, y_{1}\right) \in\left[0, \alpha_{1}\right]^{2} \\ \min \left(x_{1}, y_{1}\right), & \text { else. }\end{cases}
$$

Let now for all $\left(x_{1}, y_{1}\right) \in\left[e_{1}, 1\right]^{2}$,

$$
\begin{aligned}
S_{\psi_{e}}\left(x_{1}, y_{1}\right) & =N\left(T_{\phi_{e}}\left(N\left(x_{1}\right), N\left(y_{1}\right)\right)\right) \\
& = \begin{cases}1-\frac{1}{1-\alpha_{2}}\left(x_{1}-1\right)\left(y_{1}-1\right), & \text { if }\left(x_{1}, y_{1}\right) \in\left[\alpha_{2}, 1\right]^{2}, \\
\max \left(x_{1}, y_{1}\right), & \text { else. }\end{cases}
\end{aligned}
$$

Define $U,(\mathcal{U}(x, y))_{1}$ and $(\mathcal{U}(x, y))_{2}$ in a similar way as in Theorem 26. Then $\mathcal{U}$ is a uninorm on $\mathcal{L}^{I}$ with neutral element e for which $\mathcal{U}\left(0_{\mathcal{L}^{I}}, 1_{\mathcal{L}^{I}}\right)=\alpha$ and which is self-dual w.r.t. $\mathcal{N}_{N}$.

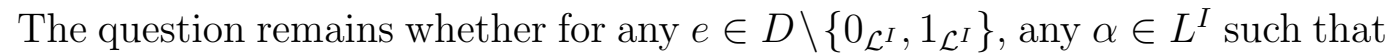
$\alpha \|_{L^{I}} e$, any t-norm $\mathcal{T}$ and any t-conorm $\mathcal{S}$ on $\mathcal{L}^{I}$, there exists a uninorm $\mathcal{U}$ on $\mathcal{L}^{I}$ with neutral element $e$ such that $\mathcal{U}\left(0_{\mathcal{L}^{I}}, 1_{\mathcal{L}^{I}}\right)=\alpha, \mathcal{T}_{\mathcal{U}}=\mathcal{T}$ and $\mathcal{S}_{\mathcal{U}}=\mathcal{S}$.

\section{Conclusion}

In this paper we have studied uninorms on the lattice $\mathcal{L}^{I}$, which is the underlying lattice of both Atanassov's intuitionistic fuzzy set theory and intervalvalued fuzzy set theory. Such uninorms $\mathcal{U}$ can be neither conjunctive nor disjunctive, in which case $\mathcal{U}\left(0_{\mathcal{L}^{I}}, 1_{\mathcal{L}^{I}}\right)$ is an element of $L^{I}$ which is incomparable to the neutral element of $\mathcal{U}$. We have investigated the value $\mathcal{U}(x, y)$ in the case that $x$ and $y$ are located in certain areas of $L^{I}$ and we have found several 
restrictions. For any value of $\alpha \in L^{I}$ which is incomparable to an arbitrary element $e$, we have constructed a uninorm $\mathcal{U}$ with neutral element $e$ and for which $\mathcal{U}\left(0_{\mathcal{L}^{I}}, 1_{\mathcal{L}^{I}}\right)=\alpha$. Such uninorms allow to model human evaluations better than uninorms on the unit interval. Uninorms on the unit interval show compensation behaviour for $x \leq e$ and $y \geq e$, with $e$ the neutral element of the uninorm, but when the extremal values 0 and 1 are inputted the uninorm can only return two possible values, namely the values 0 and 1 themselves, as the output. This is bad compensation: for example when a customer has to give an appreciation about a movie, he may give the appreciation 0 ("very bad") for the actors and the appreciation 1 ("very good") for the plot, but then it is very hard to give an overall appreciation of the movie. Uninorms on the unit interval force the customer to provide 0 or 1 as the global evaluation; this means that he has to find the movie either very good or very bad. In reality, however, his global evaluation will be more mitigated and contain a lot of uncertainty ("I find the movie not really good and also not really bad, but I don't know how to evaluate it correctly"). The solution is to use uninorms on $\mathcal{L}^{I}$ which are neither conjunctive nor disjunctive. As shown in this paper, for any value $\alpha$ which is incomparable to the neutral element, we can construct a uninorm which outputs $\alpha$ when the input values are $0_{\mathcal{L}^{I}}$ and $1_{\mathcal{L}^{I}}$. This means that uninorms on $\mathcal{L}^{I}$ are capable of modelling any global evaluation (containing any level of uncertainty) that the customer may give to the movie in our example.

\section{References}

[1] K. T. Atanassov, Intuitionistic fuzzy sets, Physica-Verlag, Heidelberg, New York, 1999.

[2] A. H. Clifford, Naturally totally ordered commutative semigroups, Amer. J. Math. 76 (1954) 631-646.

[3] B. Depaire, K. Vanhoof, G. Wets, The application of uninorms in importanceperformance analysis, in: FS'06: Proceedings of the 7th WSEAS International Conference on Fuzzy Systems, World Scientific and Engineering Academy and Society (WSEAS), Stevens Point, Wisconsin, USA, 2006.

[4] B. Depaire, K. Vanhoof, G. Wets, Expectation-performance compatibility in a customer satisfaction context modelled by means of aggregation operators, in: B. De Baets, K. Maes, R. Mesiar (eds.), Proceedings of the 4th International Summer School on Aggregation Operators, Academia Press, 2007.

[5] B. Depaire, K. Vanhoof, G. Wets, Managerial opportunities of uninormbased importance-performance analysis, WSEAS Transactions on Business and Economics 3 (3) (2007) 101-108. 
[6] G. Deschrijver, C. Cornelis, E. E. Kerre, On the representation of intuitionistic fuzzy t-norms and t-conorms, IEEE Transactions on Fuzzy Systems 12 (1) (2004) 45-61.

[7] G. Deschrijver, E. E. Kerre, On the relationship between some extensions of fuzzy set theory, Fuzzy Sets and Systems 133 (2) (2003) 227-235.

[8] G. Deschrijver, E. E. Kerre, Uninorms in $L^{*}$-fuzzy set theory, Fuzzy Sets and Systems 148 (2) (2004) 243-262.

[9] D. Dubois, On ignorance and contradiction considered as truth-values, Logic Journal of the IGPL 16 (2) (2008) 195-216.

[10] M. Eisenberg, Axiomatic theory of sets and classes, Holt, Rinehart and Winston, Inc., New York, 1971.

[11] J. C. Fodor, R. R. Yager, A. Rybalov, Structure of uninorms, International Journal of Uncertainty, Fuzziness and Knowledge-Based Systems 5 (4) (1997) $411-427$.

[12] J. A. Goguen, L-fuzzy sets, Journal of Mathematical Analysis and Applications 18 (1) (1967) 145-174.

[13] M. B. Gorzałczany, A method of inference in approximate reasoning based on interval-valued fuzzy sets, Fuzzy Sets and Systems 21 (1) (1987) 1-17.

[14] S. Jenei, A note on the ordinal sum theorem and its consequence for the construction of triangular norms, Fuzzy Sets and Systems 126 (2) (2002) 199205.

[15] C.-H. Ling, Representation of associative functions, Publ. Math. Debrecen 12 (1965) 189-212.

[16] R. Sambuc, Fonctions $\Phi$-floues. Application à l'aide au diagnostic en pathologie thyroidienne, Ph.D. thesis, Université de Marseille, France (1975).

[17] S. Saminger, On ordinal sums of triangular norms on bounded lattices, Fuzzy Sets and Systems 157 (10) (2006) 1403-1416.

[18] B. Schweizer, A. Sklar, Associative functions and abstract semigroups, Publ. Math. Debrecen 10 (1963) 69-81.

[19] K. Vanhoof, P. Pauwels, J. Dombi, T. Brijs, G. Wets, Penalty-reward analysis with uninorms: A study of customer (dis)satisfaction, in: D. Ruan, C. Chen, E. E. Kerre, G. Wets (eds.), Intelligent Data Mining: Techniques and Applications, 2005, pp. 237-252.

[20] R. R. Yager, A. Rybalov, Uninorm aggregation operators, Fuzzy Sets and Systems 80 (1) (1996) 111-120. 